Supporting information for:

\title{
An antiparasitic compound from the Medicines for Malaria Venture Pathogen Box promotes Leishmania tubulin polymerization
}

Imran Ullah ${ }^{1,2}$, Suraksha Gahalawat ${ }^{2}$, Laela M. Booshehri ${ }^{1}$, Hanspeter Niederstrasser $^{2}$, Shreoshi Majumdar $^{3}$, Christopher Leija², James M. Bradford ${ }^{1}$, Bin $\mathrm{Hu}^{2}$, Joseph M. Ready² and Dawn M. Wetzel $^{1,2^{*}}$

${ }^{1}$ Department of Pediatrics, University of Texas Southwestern Medical Center, 5323 Harry Hines Blvd, Dallas, Texas 75390, United States.

${ }^{2}$ Department of Biochemistry, University of Texas Southwestern Medical Center, 5323 Harry Hines Blvd, Dallas, Texas 75390, United States.

${ }^{3}$ Department of Biophysics, University of Texas Southwestern Medical Center, 5323 Harry Hines Blvd, Dallas, Texas 75390, United States.

*Correspondence to: dawn.wetzel@utsouthwestern.edu (DMW)

Pages S1 to S20

Figures $\mathrm{S} 1$ to $\mathrm{S} 7$

Tables S1 to S2 
Fig $\mathbf{S 1}$
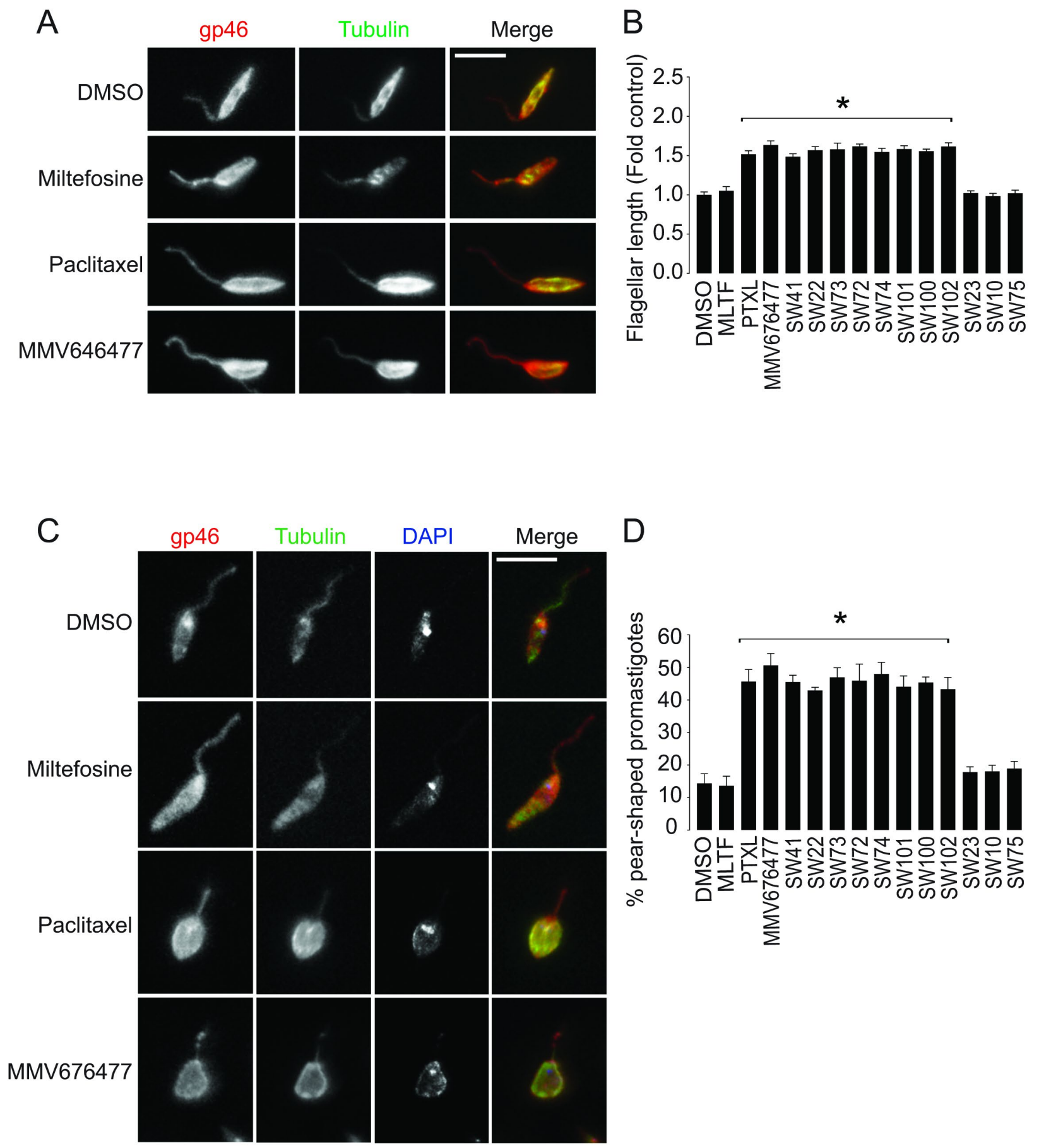

Fig S1. Morphological changes in Leishmania promastigotes treated with MMV676477 and analogs. (A) Exemplar confocal fluorescence microscopy images demonstrating flagella length in promastigotes treated for $24 \mathrm{~h}$ at the $\mathrm{EC}_{50}{ }^{72 \mathrm{~h}}$ concentration with DMSO (control), miltefosine, paclitaxel or MMV676477. Promastigotes were labeled with anti- $\alpha$-tubulin monoclonal antibody 
YL1/2 (green; Invitrogen, catalog \# MA1-80017) and anti-gp46 (membrane protein, red). Scale bar $=10 \mu \mathrm{m}$. (B) Mean flagellar length for compounds. The flagellar length for each promastigote treated as in A was measured using the gp46 channel in Image J. At least 50 parasites were analyzed per condition for three independent biological replicates (mean $+/-\mathrm{SE}$ ). ${ }^{*} p<0.05$ by ANOVA compared to DMSO control. MLTF, miltefosine; PTXL, paclitaxel. (C) Exemplar confocal fluorescence microscopy images of pear-shaped or rounded promastigotes treated for $48 \mathrm{~h}$ at the $\mathrm{EC}_{50}{ }^{72 \mathrm{~h}}$ concentration with paclitaxel or MMV676477, compared to more typically-shaped promastigotes treated with DMSO (control) and miltefosine. Labeling performed as in A; DAPI was also used to label parasite nuclei and kinetoplasts. Scale bar $=10 \mu \mathrm{m}$. (D) Percentages of pear-shaped or rounded promastigotes, defined as having a cell body with a width of $\geq 70 \%$ their length, for parasites treated as in C. At least 200 parasites were analyzed per condition per experiment for three independent biological replicates (mean $+/-\mathrm{SE}$ ). ${ }^{*} p<0.05$ by ANOVA compared to control conditions. 
Fig $\mathbf{S 2}$

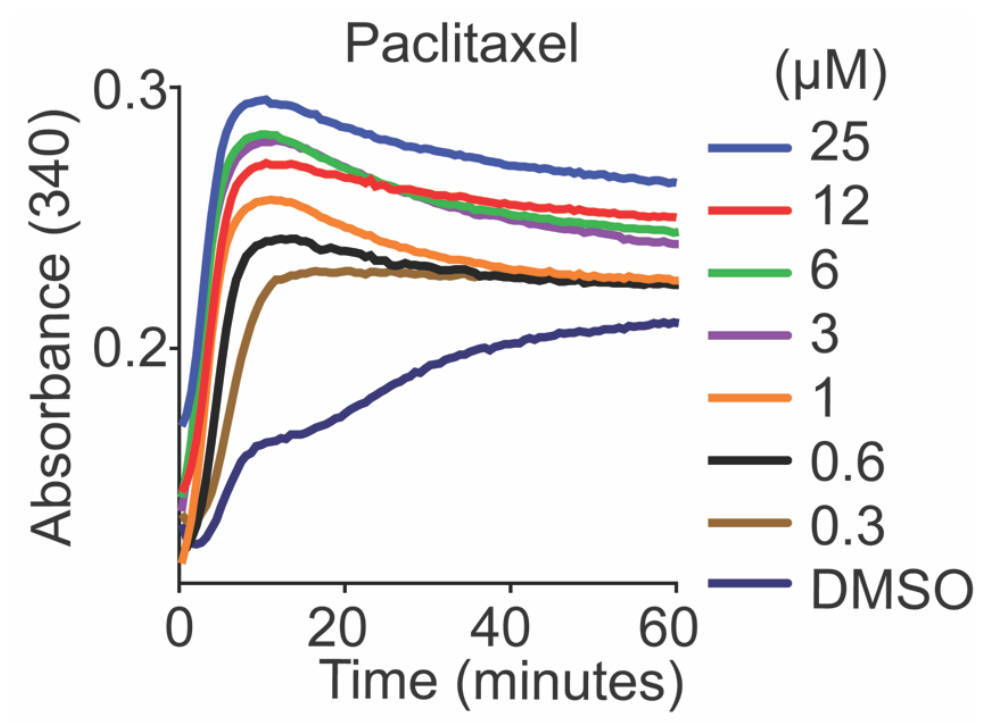

Fig S2. Effect of paclitaxel on porcine microtubule assembly in vitro. Representative turbidity curves (all concentrations in $\mu \mathrm{M}$ ) for purified porcine tubulin ( $3 \mathrm{mg} / \mathrm{mL}$ tubulin, $1 \% \mathrm{DMSO}$ ) treated with paclitaxel. For porcine tubulin: Paclitaxel $\mathrm{EC}_{50}=1.5 \pm 0.2 \mu \mathrm{M}$; Maximum absorbance (A340, $10 \%$ DMSO) $=0.36$ for paclitaxel 
Fig $\$ 3$

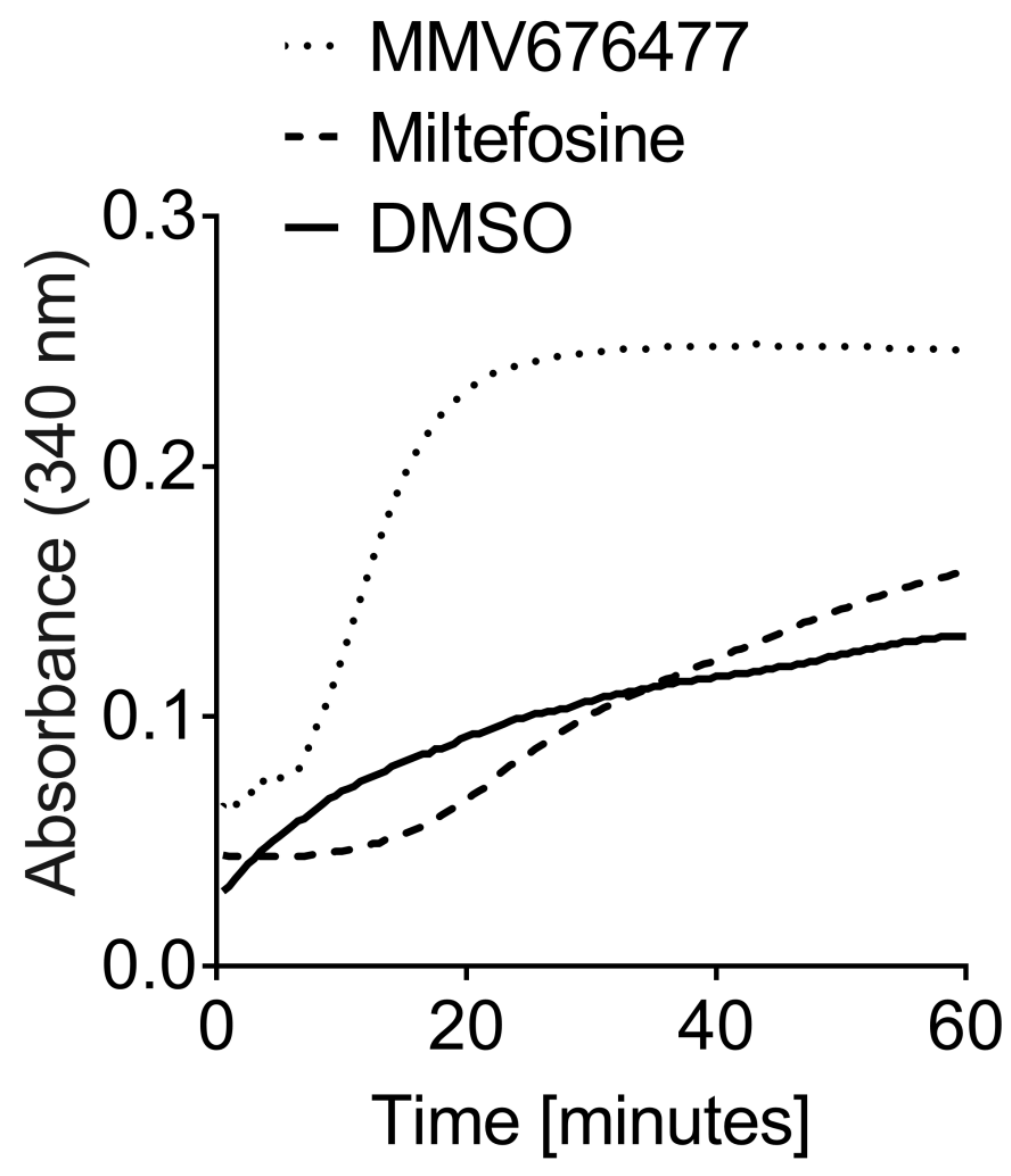

Fig S3. Effect of miltefosine on purified tubulin assembly in vitro. Miltefosine has no effect on porcine tubulin polymerization (compounds at $50 \mu \mathrm{M} ; 3 \mathrm{mg} / \mathrm{mL}$ tubulin, $1 \% \mathrm{DMSO}$ ). Shown is a representative curve from 3 separate experiments. 
Fig $\mathbf{S 4}$

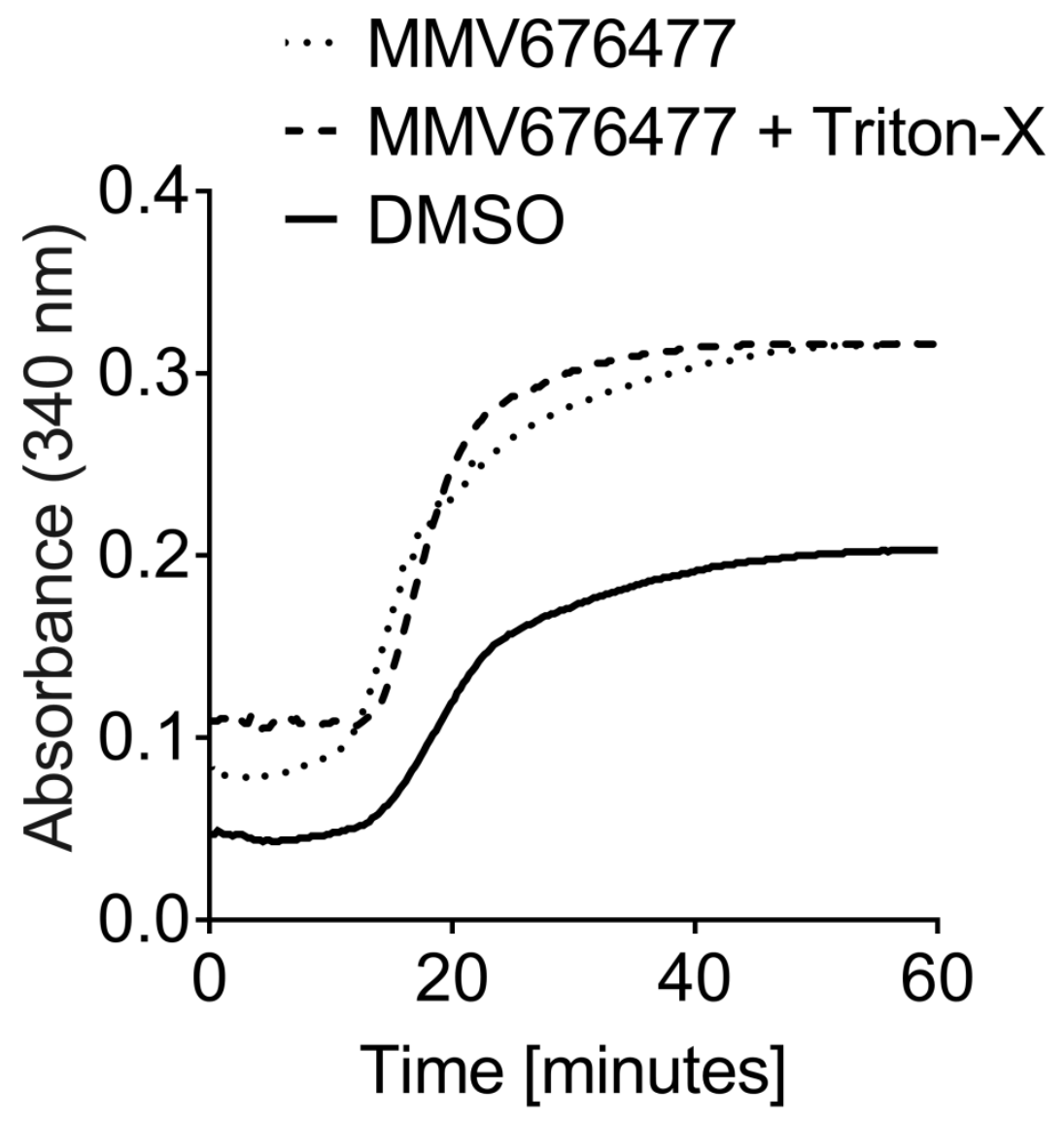

Fig S4. Relative polymerization activity of MMV676477 in presence or absence of detergent. The polymerization activity of MMV676477 is not affected by detergent $(0.01 \%$ Triton, compound at $50 \mu \mathrm{M} ; 3 \mathrm{mg} / \mathrm{mL}$ porcine tubulin, $1 \% \mathrm{DMSO}$ ). Shown is a representative curve from 3 separate experiments. 


\section{Fig S5}

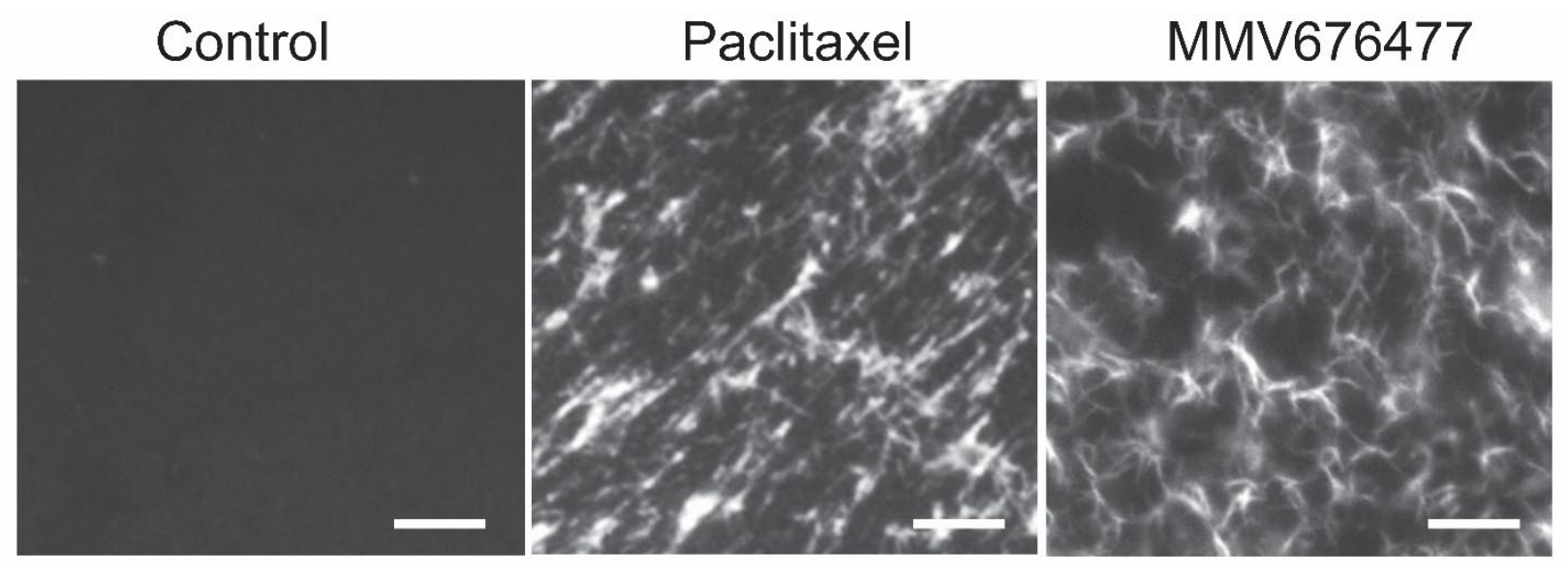

Fig S5. Fluorescent images of purified tubulin treated with Paclitaxel and MMV676477.

Fluorescent images of porcine tubulin treated with DMSO, $50 \mu \mathrm{M}$ paclitaxel and $50 \mu \mathrm{M}$ MMV676477 (40 min, 2\% DMSO). Only paclitaxel and MMV676477-treated samples contain visible tubulin under these conditions. Scale bar $=10 \mu \mathrm{m}$. 


\section{Fig $\mathbf{S 6}$}

A Competitor
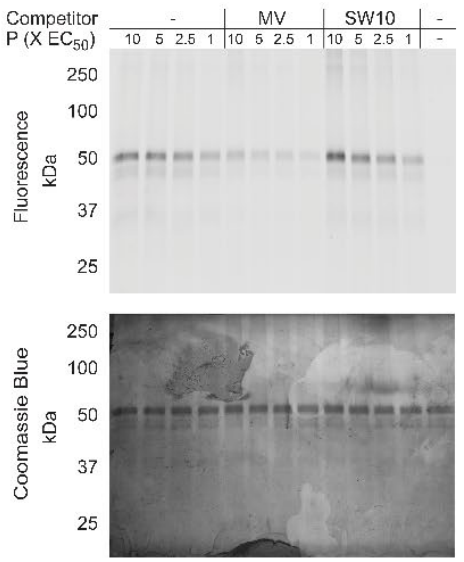

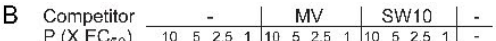
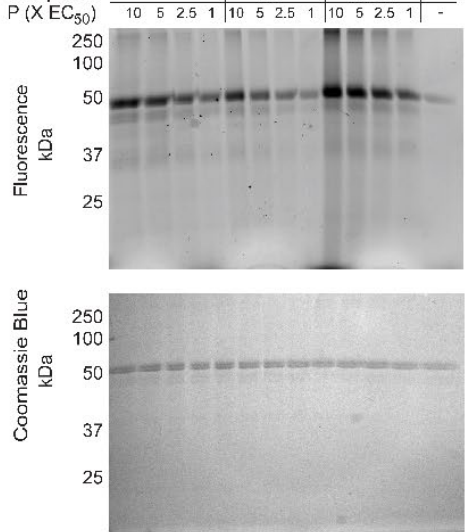

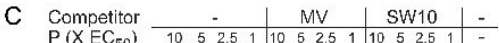
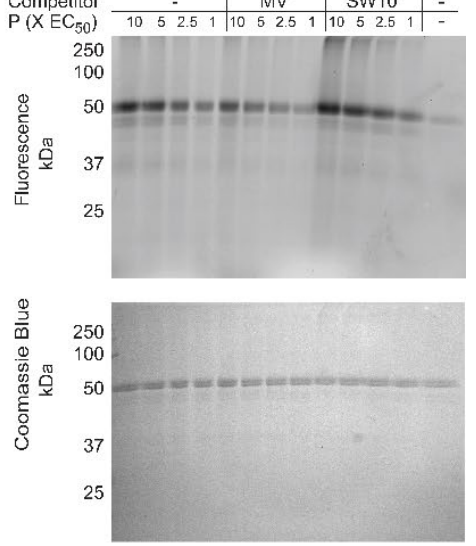

Fig S6. Full fluorescence and Coomassie blue gels from Fig 6. (A) Full fluorescence and Coomassie blue gels from cropped image shown in Fig 6. (B-C) Remaining full fluorescence and Coomassie gels used for densiometric analyses. Densitometric analyses are shown in Fig 6B. Note that samples in A were run using a $4-12 \%$ gel (BioRad pre-cast) while those in $B$ and $C$ were run using a $12 \%$ gel. 
Fig $\mathbf{S 7}$

A

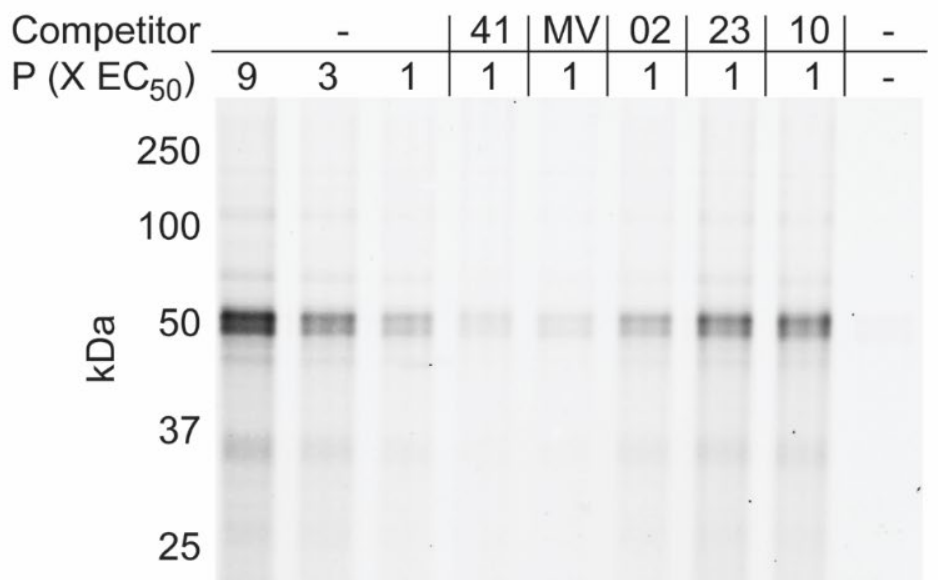

Fluorescence

B

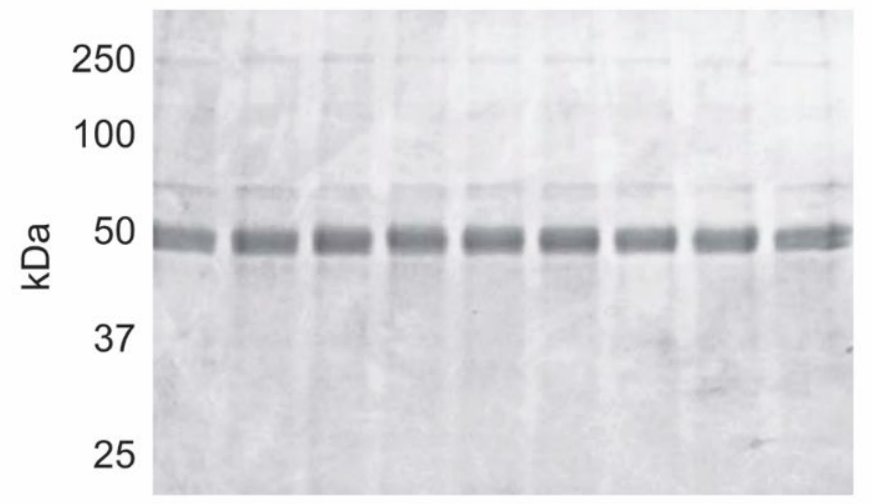

Coomassie Blue

Fig S7. Competition-sensitive fluorescent binding of MMV676477 analogs to tubulin and additional proteins. Following treatment with the SW22 probe ("P") in the presence or absence of competitors, L. tarentolae tubulin (see methods for details) was subjected to UV crosslinking. Alexa Fluor 532 azide dye was then conjugated to the probe via CuAAC. (A) Fluorescence imaging of dye-labeled tubulin samples, \pm competition by MMV676477 and a panel of analogs of varying potency. Competing compounds were added at 100X the probe concentration (e.g. 1X probe $\mathrm{EC}_{50}=156 \mathrm{nM}$; competitors added at $\left.15.6 \mu \mathrm{M}\right) . \mathrm{EC}_{50}{ }^{72 \mathrm{~h}}$ data and structures in table 4. (B) Coomassie blue staining of the gel in $(A)$ is used as a loading control. 


\section{Table S1}

Table S1. Screening of the MMV Pathogen Box. MMV Pathogen Box compounds were screened at $5 \mu \mathrm{M}$ and $1 \mu \mathrm{M}$ against $L$. amazonensis axenic amastigotes. Relative fluorescence intensity (\%) is shown. Red values indicate the most potent compounds at $1 \mu \mathrm{M}$; blue values indicate the least potent compounds at $1 \mu \mathrm{M}$.

Compound ID Disease Set

\begin{tabular}{|c|c|c|c|c|c|}
\hline & & $5 \mu \mathrm{M}$ & SD & $1 \mu \mathrm{M}$ & SD \\
\hline MMV676477 & $\begin{array}{l}\text { TUBERCULOSIS } \\
\text { REFERENCE }\end{array}$ & -0.5 & 0.0 & -0.5 & 0.1 \\
\hline MMV689480 & COMPOUNDS & -3.0 & 2.1 & -0.5 & 0.9 \\
\hline MMV676412 & TUBERCULOSIS & -1.6 & 0.4 & -0.5 & 0.3 \\
\hline MMV688262 & TUBERCULOSIS & -0.8 & 1.8 & -0.5 & 1.7 \\
\hline MMV688372 & $\begin{array}{l}\text { KINETOPLASTIDS } \\
\text { REFERENCE }\end{array}$ & 0.1 & 0.0 & 0.6 & 1.4 \\
\hline MMV688978 & COMPOUNDS & -0.1 & 2.7 & 0.8 & 1.2 \\
\hline MMV011903 & MALARIA & -1.5 & 0.2 & 3.5 & 0.6 \\
\hline MMV652003 & KINETOPLASTIDS & -5.1 & 1.9 & 4.4 & 11.5 \\
\hline MMV595321 & KINETOPLASTIDS & -5.1 & 1.9 & 4.4 & 11.5 \\
\hline MMV090930 & $\begin{array}{l}\text { TUBERCULOSIS } \\
\text { REFERENCE }\end{array}$ & -0.2 & 1.5 & 7.2 & 3.7 \\
\hline MMV688991 & $\begin{array}{l}\text { COMPOUNDS } \\
\text { LYMPHATIC }\end{array}$ & 8.5 & 1.0 & 61.8 & 9.2 \\
\hline MMV687775 & $\begin{array}{l}\text { FILARIASIS } \\
\text { REFERENCE }\end{array}$ & 76.3 & 13.4 & 71.4 & 5.6 \\
\hline MMV688994 & COMPOUNDS & 76.3 & 13.4 & 71.4 & 5.6 \\
\hline MMV200748 & TUBERCULOSIS & 101.0 & 4.2 & 71.7 & 23.3 \\
\hline MMV688853 & CRYPTOSPORIDIOSIS & 77.1 & 11.3 & 72.1 & 1.4 \\
\hline MMV688417 & TOXOPLASMOSIS & 29.0 & 3.2 & 72.1 & 9.2 \\
\hline MMV690027 & KINETOPLASTIDS & 51.6 & 6.7 & 72.7 & 5.0 \\
\hline MMV688415 & KINETOPLASTIDS & 60.5 & 2.8 & 73.5 & 9.6 \\
\hline MMV676406 & TUBERCULOSIS & 63.2 & 3.7 & 73.9 & 5.0 \\
\hline MMV688755 & TUBERCULOSIS & 39.5 & 5.1 & 74.0 & 8.1 \\
\hline MMV688797 & KINETOPLASTIDS & 42.3 & 1.4 & 74.5 & 3.8 \\
\hline MMV688942 & KINETOPLASTIDS & 80.2 & 7.6 & 74.5 & 9.5 \\
\hline
\end{tabular}

Relative fluorescence intensity (\%) 


\begin{tabular}{|c|c|c|c|c|c|}
\hline MMV688467 & KINETOPLASTIDS & 66.3 & 22.5 & 74.8 & 27.6 \\
\hline MMV690028 & KINETOPLASTIDS & 43.9 & 5.9 & 74.9 & 4.6 \\
\hline MMV689244 & KINETOPLASTIDS & 49.1 & 33.1 & 74.9 & 25.2 \\
\hline MMV023949 & MALARIA & 56.3 & 3.3 & 75.0 & 5.6 \\
\hline MMV011765 & MALARIA & 95.6 & 8.8 & 75.0 & 3.0 \\
\hline MMV560185 & MALARIA & 75.9 & 12.3 & 75.1 & 5.4 \\
\hline MMV062221 & MALARIA & 63.1 & 9.9 & 75.2 & 15.1 \\
\hline MMV688943 & KINETOPLASTIDS & 47.0 & 1.6 & 75.4 & 0.2 \\
\hline MMV676442 & MALARIA & 92.2 & 3.2 & 75.4 & 19.6 \\
\hline MMV688854 & CRYPTOSPORIDIOSIS & 86.0 & 1.6 & 75.9 & 11.4 \\
\hline MMV495543 & TUBERCULOSIS & 95.3 & 4.8 & 76.9 & 17.2 \\
\hline MMV676388 & TUBERCULOSIS & 45.7 & 3.8 & 77.1 & 18.4 \\
\hline MMV000858 & MALARIA & -2.2 & 0.7 & 77.3 & 4.7 \\
\hline MMV687729 & TUBERCULOSIS & 81.3 & 1.6 & 77.6 & 7.3 \\
\hline MMV689243 & KINETOPLASTIDS & 92.3 & 1.5 & 77.7 & 0.6 \\
\hline MMV676386 & TUBERCULOSIS & 54.3 & 5.6 & 77.7 & 14.2 \\
\hline MMV688552 & $\begin{array}{l}\text { SCHISTOSOMIASIS } \\
\text { REFERENCE }\end{array}$ & 80.2 & 10.1 & 78.7 & 8.4 \\
\hline MMV002816 & COMPOUNDS & 97.6 & 1.9 & 78.8 & 0.7 \\
\hline MMV021013 & TUBERCULOSIS & 97.3 & 2.2 & 78.9 & 1.9 \\
\hline MMV688543 & DENGUE & 61.9 & 1.7 & 79.1 & 3.6 \\
\hline MMV676384 & TUBERCULOSIS & 103.1 & 6.7 & 79.1 & 13.1 \\
\hline MMV675996 & ONCHOCERCIASIS & 99.7 & 1.7 & 79.2 & 3.5 \\
\hline MMV188296 & KINETOPLASTIDS & 64.6 & 9.3 & 79.3 & 5.9 \\
\hline MMV032995 & MALARIA & 78.8 & 1.8 & 79.3 & 1.8 \\
\hline MMV676358 & MALARIA & 89.1 & 5.6 & 79.4 & 7.5 \\
\hline MMV020289 & MALARIA & 98.2 & 2.4 & 79.4 & 13.3 \\
\hline MMV028694 & MALARIA & 92.4 & 4.9 & 79.5 & 5.8 \\
\hline MMV659010 & KINETOPLASTIDS & 62.1 & 2.5 & 79.6 & 1.6 \\
\hline MMV688939 & TUBERCULOSIS & 77.2 & 14.1 & 79.8 & 15.1 \\
\hline MMV024937 & $\begin{array}{l}\text { MALARIA } \\
\text { LYMPHATIC }\end{array}$ & 94.9 & 3.6 & 79.9 & 1.8 \\
\hline MMV676492 & FILARIASIS & 84.7 & 0.4 & 80.4 & 2.6 \\
\hline MMV611037 & TUBERCULOSIS & 99.5 & 4.7 & 80.6 & 2.0 \\
\hline MMV688766 & SCHISTOSOMIASIS & 82.7 & 3.6 & 80.8 & 3.2 \\
\hline MMV019742 & MALARIA & 77.7 & 2.5 & 80.8 & 3.4 \\
\hline MMV023985 & MALARIA & 83.2 & 5.4 & 81.1 & 6.8 \\
\hline MMV007471 & MALARIA & 74.3 & 5.6 & 81.2 & 2.8 \\
\hline MMV676050 & CRYPTOSPORIDIOSIS & 80.8 & 2.4 & 81.4 & 4.8 \\
\hline MMV687765 & TUBERCULOSIS & 82.9 & 1.7 & 81.5 & 6.2 \\
\hline MMV676380 & MALARIA & 101.1 & 2.6 & 81.5 & 4.8 \\
\hline MMV1198433 & SCHISTOSOMIASIS & 84.1 & 8.3 & 81.6 & 4.5 \\
\hline MMV001561 & KINETOPLASTIDS & 91.9 & 2.7 & 81.6 & 15.5 \\
\hline MMV676057 & KINETOPLASTIDS & 101.7 & 15.9 & 81.6 & 9.0 \\
\hline MMV689028 & KINETOPLASTIDS & 68.8 & 2.9 & 81.6 & 4.7 \\
\hline
\end{tabular}




\begin{tabular}{|c|c|c|c|c|c|}
\hline MMV676555 & TUBERCULOSIS & 69.6 & 3.1 & 81.8 & 2.8 \\
\hline MMV016838 & MALARIA & 65.9 & 9.7 & 81.9 & 15.0 \\
\hline MMV676476 & TUBERCULOSIS & 99.3 & 0.9 & 82.0 & 9.3 \\
\hline MMV676472 & TUBERCULOSIS & -1.8 & 0.7 & 82.1 & 2.9 \\
\hline MMV688795 & KINETOPLASTIDS & 93.6 & 7.9 & 82.2 & 3.4 \\
\hline MMV676524 & TUBERCULOSIS & 94.6 & 1.0 & 82.3 & 3.6 \\
\hline MMV688345 & TOXOPLASMOSIS & 82.5 & 3.6 & 82.3 & 2.6 \\
\hline MMV1019989 & MALARIA & 103.7 & 5.6 & 82.3 & 2.3 \\
\hline MMV461553 & TUBERCULOSIS & 66.4 & 8.6 & 82.4 & 7.3 \\
\hline MMV393995 & TUBERCULOSIS & 26.0 & 8.3 & 82.4 & 3.5 \\
\hline MMV023233 & MALARIA & 65.2 & 4.2 & 82.6 & 0.5 \\
\hline MMV675995 & ONCHOCERCIASIS & 42.7 & 3.5 & 82.7 & 0.2 \\
\hline MMV1030799 & MALARIA & 83.8 & 6.5 & 82.7 & 3.5 \\
\hline MMV688471 & $\begin{array}{l}\text { TOXOPLASMOSIS } \\
\text { REFERENCE }\end{array}$ & -0.7 & 1.2 & 82.7 & 4.2 \\
\hline MMV688774 & $\begin{array}{l}\text { COMPOUNDS } \\
\text { REFERENCE }\end{array}$ & 75.3 & 1.8 & 82.7 & 2.7 \\
\hline MMV688990 & COMPOUNDS & 88.4 & 7.4 & 82.9 & 10.8 \\
\hline MMV690102 & KINETOPLASTIDS & 91.7 & 10.9 & 82.9 & 2.3 \\
\hline MMV687700 & TUBERCULOSIS & 7.2 & 0.6 & 83.0 & 0.9 \\
\hline MMV676431 & TUBERCULOSIS & 111.6 & 1.6 & 83.2 & 2.3 \\
\hline MMV675998 & KINETOPLASTIDS & 78.6 & 13.5 & 83.2 & 11.6 \\
\hline MMV688330 & TOXOPLASMOSIS & 100.9 & 7.0 & 83.2 & 2.7 \\
\hline MMV020320 & MALARIA & 94.1 & 3.7 & 83.3 & 3.6 \\
\hline MMV006239 & MALARIA & 89.1 & 3.2 & 83.3 & 13.2 \\
\hline MMV020388 & MALARIA & 85.1 & 5.7 & 83.4 & 4.1 \\
\hline MMV688771 & SCHISTOSOMIASIS & 78.2 & 1.4 & 83.6 & 2.6 \\
\hline MMV020165 & MALARIA & 83.8 & 5.3 & 83.8 & 6.0 \\
\hline MMV688889 & TUBERCULOSIS & 102.3 & 5.5 & 83.8 & 4.9 \\
\hline MMV007803 & MALARIA & 76.4 & 12.7 & 84.1 & 13.1 \\
\hline MMV687747 & TUBERCULOSIS & 76.3 & 25.9 & 84.1 & 11.1 \\
\hline MMV085230 & MALARIA & 71.1 & 1.5 & 84.2 & 4.6 \\
\hline MMV392832 & MALARIA & 93.9 & 5.3 & 84.2 & 1.9 \\
\hline MMV019838 & MALARIA & 104.3 & 5.7 & 84.3 & 5.3 \\
\hline MMV637229 & TRICHURIASIS & 26.4 & 10.6 & 84.3 & 19.3 \\
\hline MMV026550 & MALARIA & 80.3 & 2.8 & 84.3 & 2.7 \\
\hline MMV146306 & TUBERCULOSIS & 96.8 & 8.8 & 84.4 & 3.5 \\
\hline MMV019189 & MALARIA & 101.5 & 13.9 & 84.4 & 8.7 \\
\hline MMV676588 & TUBERCULOSIS & 70.9 & 6.9 & 84.4 & 12.4 \\
\hline MMV667494 & MALARIA & 82.2 & 4.5 & 84.5 & 15.7 \\
\hline MMV676350 & MALARIA & 110.0 & 1.7 & 84.5 & 1.2 \\
\hline MMV687749 & TUBERCULOSIS & 98.6 & 7.9 & 84.7 & 11.3 \\
\hline MMV272144 & TUBERCULOSIS & 94.2 & 2.0 & 84.7 & 2.9 \\
\hline MMV030734 & MALARIA & 59.9 & 13.4 & 84.7 & 1.9 \\
\hline MMV688845 & TUBERCULOSIS & 101.3 & 1.8 & 84.8 & 1.4 \\
\hline
\end{tabular}




\begin{tabular}{|c|c|c|c|c|c|}
\hline MMV687243 & TUBERCULOSIS & 56.2 & 1.7 & 84.8 & 6.9 \\
\hline MMV020710 & MALARIA & 89.2 & 2.8 & 84.8 & 3.7 \\
\hline MMV687251 & TUBERCULOSIS & 84.9 & 6.7 & 84.9 & 2.0 \\
\hline MMV153413 & TUBERCULOSIS & 18.7 & 13.7 & 84.9 & 3.5 \\
\hline MMV676162 & KINETOPLASTIDS & -0.4 & 0.1 & 85.1 & 1.2 \\
\hline MMV688938 & $\begin{array}{l}\text { TUBERCULOSIS } \\
\text { REFERENCE }\end{array}$ & 49.4 & 9.0 & 85.1 & 5.0 \\
\hline MMV687801 & COMPOUNDS & 109.1 & 7.0 & 85.3 & 3.4 \\
\hline MMV676270 & MALARIA & 90.9 & 2.0 & 85.3 & 6.7 \\
\hline MMV688557 & TUBERCULOSIS & 92.3 & 6.6 & 85.3 & 5.6 \\
\hline MMV010764 & MALARIA & 90.5 & 10.1 & 85.4 & 6.3 \\
\hline MMV676204 & ONCHOCERCIASIS & 87.4 & 10.6 & 85.6 & 17.8 \\
\hline MMV676599 & CRYPTOSPORIDIOSIS & 84.0 & 4.1 & 85.6 & 3.7 \\
\hline MMV676470 & TUBERCULOSIS & 89.7 & 7.2 & 85.8 & 8.3 \\
\hline MMV020520 & MALARIA & 105.7 & 7.4 & 85.8 & 9.0 \\
\hline MMV659004 & KINETOPLASTIDS & 85.4 & 4.4 & 86.0 & 3.3 \\
\hline MMV024397 & MALARIA & 52.3 & 6.1 & 86.0 & 5.9 \\
\hline MMV689437 & KINETOPLASTIDS & 90.9 & 7.6 & 86.0 & 12.0 \\
\hline MMV688509 & TOXOPLASMOSIS & 90.0 & 3.3 & 86.0 & 2.1 \\
\hline MMV687730 & TUBERCULOSIS & 68.9 & 1.4 & 86.0 & 3.4 \\
\hline MMV054312 & TUBERCULOSIS & 90.4 & 1.1 & 86.1 & 1.2 \\
\hline MMV676501 & TUBERCULOSIS & 94.8 & 4.0 & 86.2 & 3.9 \\
\hline MMV011229 & $\begin{array}{l}\text { MALARIA } \\
\text { REFERENCE }\end{array}$ & 2.9 & 2.3 & 86.2 & 7.0 \\
\hline MMV689000 & COMPOUNDS & 108.8 & 4.8 & 86.2 & 9.3 \\
\hline MMV688122 & TUBERCULOSIS & 102.2 & 3.5 & 86.4 & 4.9 \\
\hline MMV676571 & TUBERCULOSIS & 97.0 & 3.1 & 86.4 & 3.8 \\
\hline MMV688472 & TOXOPLASMOSIS & 48.3 & 12.4 & 86.5 & 5.2 \\
\hline MMV023227 & MALARIA & 79.3 & 10.4 & 86.5 & 10.6 \\
\hline MMV407834 & MALARIA & 109.9 & 2.3 & 86.6 & 1.4 \\
\hline MMV688273 & KINETOPLASTIDS & 95.9 & 1.8 & 86.8 & 2.8 \\
\hline MMV688756 & TUBERCULOSIS & 95.8 & 14.1 & 86.8 & 3.0 \\
\hline MMV687138 & TUBERCULOSIS & 106.1 & 5.4 & 86.8 & 17.1 \\
\hline MMV012074 & TUBERCULOSIS & 84.3 & 2.7 & 86.8 & 4.5 \\
\hline MMV688955 & TOXOPLASMOSIS & 87.4 & 3.0 & 87.0 & 3.8 \\
\hline MMV024195 & MALARIA & 84.6 & 12.0 & 87.0 & 4.1 \\
\hline MMV676536 & SCHISTOSOMIASIS & 90.8 & 19.8 & 87.0 & 8.3 \\
\hline MMV688270 & SCHISTOSOMIASIS & 85.1 & 3.0 & 87.0 & 1.0 \\
\hline MMV024406 & MALARIA & 108.3 & 7.0 & 87.1 & 4.7 \\
\hline MMV026468 & MALARIA & 98.3 & 8.8 & 87.1 & 6.6 \\
\hline MMV687170 & TUBERCULOSIS & 105.2 & 5.8 & 87.1 & 5.3 \\
\hline MMV084864 & MALARIA & 82.8 & 2.5 & 87.1 & 3.8 \\
\hline MMV009054 & MALARIA & 103.9 & 9.6 & 87.2 & 8.9 \\
\hline MMV675997 & KINETOPLASTIDS & 82.3 & 2.4 & 87.2 & 5.9 \\
\hline MMV688466 & TUBERCULOSIS & 98.2 & 2.8 & 87.3 & 2.0 \\
\hline
\end{tabular}




\begin{tabular}{|c|c|c|c|c|c|}
\hline MMV688514 & $\begin{array}{l}\text { KINETOPLASTIDS } \\
\text { REFERENCE }\end{array}$ & 98.9 & 2.2 & 87.4 & 1.0 \\
\hline MMV687796 & COMPOUNDS & 101.3 & 4.1 & 87.4 & 8.7 \\
\hline MMV020537 & MALARIA & 101.5 & 1.3 & 87.5 & 1.3 \\
\hline MMV688846 & TUBERCULOSIS & 89.6 & 7.2 & 87.6 & 6.9 \\
\hline MMV019993 & MALARIA & 93.4 & 2.2 & 87.7 & 2.2 \\
\hline MMV008439 & MALARIA & 105.7 & 4.7 & 87.7 & 10.4 \\
\hline MMV085499 & MALARIA & 98.8 & 0.6 & 87.7 & 2.0 \\
\hline MMV688327 & TUBERCULOSIS & 95.2 & 5.9 & 87.8 & 0.2 \\
\hline MMV687180 & TUBERCULOSIS & 101.3 & 4.7 & 87.8 & 1.0 \\
\hline MMV023370 & MALARIA & 83.6 & 6.2 & 87.8 & 6.6 \\
\hline MMV407539 & $\begin{array}{l}\text { WOLBACHIA LF } \\
\text { REFERENCE }\end{array}$ & 90.9 & 2.6 & 88.0 & 6.1 \\
\hline MMV002529 & $\begin{array}{l}\text { COMPOUNDS } \\
\text { REFERENCE }\end{array}$ & 86.0 & 20.7 & 88.0 & 11.0 \\
\hline MMV688773 & COMPOUNDS & 82.2 & 1.9 & 88.1 & 2.5 \\
\hline MMV023183 & MALARIA & 88.9 & 4.8 & 88.2 & 4.9 \\
\hline MMV676269 & $\begin{array}{l}\text { MALARIA } \\
\text { REFERENCE }\end{array}$ & 106.9 & 5.2 & 88.2 & 6.0 \\
\hline MMV637953 & COMPOUNDS & 92.4 & 7.8 & 88.4 & 5.9 \\
\hline MMV688371 & KINETOPLASTIDS & 90.4 & 1.4 & 88.4 & 1.4 \\
\hline MMV676377 & TUBERCULOSIS & 96.5 & 1.5 & 88.5 & 1.3 \\
\hline MMV687699 & TUBERCULOSIS & 94.0 & 1.7 & 88.6 & 2.8 \\
\hline MMV161996 & TUBERCULOSIS & 86.3 & 2.8 & 88.6 & 11.9 \\
\hline MMV676383 & TUBERCULOSIS & 86.4 & 11.7 & 88.6 & 9.9 \\
\hline MMV676526 & TUBERCULOSIS & 90.3 & 7.2 & 88.6 & 8.9 \\
\hline MMV675993 & CRYPTOSPORIDIOSIS & 65.2 & 17.7 & 88.6 & 14.2 \\
\hline MMV1236379 & KINETOPLASTIDS & 89.6 & 4.8 & 88.7 & 2.4 \\
\hline MMV688179 & KINETOPLASTIDS & 101.8 & 1.9 & 88.7 & 1.8 \\
\hline MMV676053 & CRYPTOSPORIDIOSIS & 96.3 & 2.9 & 88.8 & 3.5 \\
\hline MMV676589 & TUBERCULOSIS & 87.8 & 2.5 & 88.8 & 5.0 \\
\hline MMV688754 & KINETOPLASTIDS & 64.0 & 8.9 & 88.8 & 3.1 \\
\hline MMV688704 & TOXOPLASMOSIS & 68.5 & 2.1 & 88.9 & 6.1 \\
\hline MMV687145 & TUBERCULOSIS & 75.3 & 8.1 & 89.0 & 1.5 \\
\hline MMV676468 & TUBERCULOSIS & 98.5 & 4.6 & 89.1 & 7.1 \\
\hline MMV023969 & TUBERCULOSIS & 105.1 & 11.5 & 89.1 & 8.5 \\
\hline MMV676584 & TUBERCULOSIS & 91.9 & 2.1 & 89.1 & 1.8 \\
\hline MMV688703 & TOXOPLASMOSIS & 96.1 & 7.8 & 89.2 & 9.5 \\
\hline MMV688411 & TOXOPLASMOSIS & 94.7 & 4.1 & 89.3 & 5.0 \\
\hline MMV688508 & TUBERCULOSIS & 108.5 & 1.6 & 89.3 & 2.9 \\
\hline MMV063404 & TUBERCULOSIS & 96.5 & 7.1 & 89.4 & 2.5 \\
\hline MMV687703 & TUBERCULOSIS & 90.9 & 7.8 & 89.6 & 7.4 \\
\hline MMV688283 & KINETOPLASTIDS & 91.7 & 2.2 & 89.6 & 1.8 \\
\hline MMV019551 & MALARIA & 96.7 & 3.8 & 89.6 & 1.4 \\
\hline MMV676008 & KINETOPLASTIDS & 82.0 & 0.6 & 89.7 & 1.5 \\
\hline
\end{tabular}




\begin{tabular}{|c|c|c|c|c|c|}
\hline MMV688352 & DENGUE & 91.4 & 9.3 & 89.7 & 9.4 \\
\hline MMV002817 & ONCHOCERCIASIS & 107.9 & 3.8 & 89.7 & 4.2 \\
\hline MMV688958 & KINETOPLASTIDS & 4.6 & 2.0 & 89.8 & 2.9 \\
\hline MMV687239 & TUBERCULOSIS & 76.5 & 2.0 & 89.8 & 7.0 \\
\hline MMV026313 & MALARIA & 99.5 & 3.2 & 89.8 & 2.3 \\
\hline MMV1088520 & MALARIA & 105.1 & 8.1 & 89.8 & 6.6 \\
\hline MMV676159 & KINETOPLASTIDS & 46.3 & 16.8 & 89.8 & 3.6 \\
\hline MMV687813 & TUBERCULOSIS & 89.1 & 6.1 & 89.9 & 2.0 \\
\hline MMV024443 & MALARIA & 102.1 & 1.3 & 89.9 & 0.9 \\
\hline MMV676478 & TUBERCULOSIS & 95.3 & 12.7 & 89.9 & 2.4 \\
\hline MMV675994 & CRYPTOSPORIDIOSIS & 92.0 & 1.8 & 90.0 & 1.0 \\
\hline MMV658988 & KINETOPLASTIDS & 87.2 & 5.8 & 90.0 & 3.8 \\
\hline MMV023860 & MALARIA & 84.2 & 5.7 & 90.1 & 6.3 \\
\hline MMV688547 & KINETOPLASTIDS & 97.7 & 3.9 & 90.1 & 9.1 \\
\hline MMV003270 & HOOKWORM & 101.1 & 2.3 & 90.1 & 2.7 \\
\hline MMV676182 & CRYPTOSPORIDIOSIS & 91.7 & 6.6 & 90.2 & 5.5 \\
\hline MMV688124 & TUBERCULOSIS & 95.8 & 4.8 & 90.2 & 5.8 \\
\hline MMV687807 & TUBERCULOSIS & 94.9 & 5.8 & 90.2 & 6.0 \\
\hline MMV676602 & KINETOPLASTIDS & 88.2 & 5.3 & 90.4 & 1.4 \\
\hline MMV687794 & $\begin{array}{l}\text { MALARIA } \\
\text { REFERENCE }\end{array}$ & 99.6 & 5.1 & 90.4 & 5.9 \\
\hline MMV001499 & COMPOUNDS & 70.9 & 3.1 & 90.4 & 5.2 \\
\hline MMV676509 & TUBERCULOSIS & 93.6 & 0.1 & 90.4 & 7.2 \\
\hline MMV687248 & TUBERCULOSIS & 65.8 & 2.9 & 90.5 & 1.8 \\
\hline MMV688798 & KINETOPLASTIDS & 94.9 & 3.0 & 90.5 & 3.4 \\
\hline MMV019790 & MALARIA & 71.6 & 1.0 & 90.5 & 3.2 \\
\hline MMV688844 & TUBERCULOSIS & 91.2 & 11.6 & 90.5 & 9.2 \\
\hline MMV688313 & SCHISTOSOMIASIS & 92.2 & 1.3 & 90.5 & 2.4 \\
\hline MMV021660 & TUBERCULOSIS & 78.1 & 5.4 & 90.5 & 6.6 \\
\hline MMV019721 & MALARIA & 95.6 & 4.3 & 90.5 & 1.3 \\
\hline MMV634140 & MALARIA & 83.6 & 12.0 & 90.6 & 4.9 \\
\hline MMV689709 & KINETOPLASTIDS & 103.8 & 6.4 & 90.7 & 2.7 \\
\hline MMV676449 & TUBERCULOSIS & 95.0 & 3.2 & 90.8 & 6.1 \\
\hline MMV687189 & TUBERCULOSIS & 74.0 & 18.7 & 90.9 & 17.0 \\
\hline MMV688762 & SCHISTOSOMIASIS & 90.5 & 7.6 & 91.0 & 4.7 \\
\hline MMV658993 & KINETOPLASTIDS & 94.5 & 7.9 & 91.0 & 6.5 \\
\hline MMV687146 & TUBERCULOSIS & 93.0 & 4.6 & 91.0 & 6.2 \\
\hline MMV004168 & KINETOPLASTIDS & 90.4 & 8.7 & 91.0 & 5.5 \\
\hline MMV688360 & KINETOPLASTIDS & 110.1 & 4.6 & 91.0 & 1.5 \\
\hline MMV020517 & MALARIA & 92.6 & 10.5 & 91.1 & 12.4 \\
\hline MMV026356 & MALARIA & 95.3 & 3.7 & 91.1 & 3.1 \\
\hline MMV687254 & TUBERCULOSIS & 121.5 & 8.9 & 91.1 & 5.3 \\
\hline MMV085071 & MALARIA & 89.1 & 5.5 & 91.1 & 4.2 \\
\hline MMV1029203 & MALARIA & 88.4 & 11.6 & 91.2 & 9.6 \\
\hline MMV688125 & TUBERCULOSIS & 99.1 & 1.3 & 91.3 & 1.8 \\
\hline
\end{tabular}




\begin{tabular}{|c|c|c|c|c|c|}
\hline & REFERENCE & & & & \\
\hline MMV688775 & COMPOUNDS & 100.3 & 3.3 & 91.3 & 3.6 \\
\hline MMV688550 & KINETOPLASTIDS & 110.3 & 1.3 & 91.4 & 7.5 \\
\hline MMV688891 & TUBERCULOSIS & 86.0 & 3.0 & 91.4 & 1.5 \\
\hline MMV675969 & ONCHOCERCIASIS & 96.1 & 4.1 & 91.5 & 5.2 \\
\hline MMV688410 & KINETOPLASTIDS & 90.4 & 4.6 & 91.5 & 4.7 \\
\hline MMV024311 & TUBERCULOSIS & 100.8 & 3.9 & 91.6 & 3.2 \\
\hline MMV020591 & MALARIA & 96.0 & 3.0 & 91.6 & 4.8 \\
\hline MMV021375 & MALARIA & 85.9 & 3.0 & 91.8 & 4.7 \\
\hline MMV687696 & TUBERCULOSIS & 95.1 & 6.2 & 91.8 & 1.0 \\
\hline MMV676554 & TUBERCULOSIS & 102.9 & 3.8 & 91.9 & 3.4 \\
\hline MMV689029 & KINETOPLASTIDS & 113.0 & 4.5 & 92.0 & 8.6 \\
\hline MMV688274 & KINETOPLASTIDS & 93.6 & 4.7 & 92.1 & 4.5 \\
\hline MMV688921 & DENGUE & 97.5 & 4.0 & 92.1 & 8.0 \\
\hline MMV688361 & $\begin{array}{l}\text { KINETOPLASTIDS } \\
\text { REFERENCE }\end{array}$ & 92.6 & 9.3 & 92.1 & 6.4 \\
\hline MMV001625 & COMPOUNDS & 98.3 & 7.1 & 92.1 & 5.1 \\
\hline MMV228911 & TUBERCULOSIS & 96.9 & 2.3 & 92.1 & 4.9 \\
\hline MMV688852 & TOXOPLASMOSIS & 52.2 & 2.5 & 92.2 & 10.9 \\
\hline MMV006833 & MALARIA & 99.8 & 3.9 & 92.2 & 1.1 \\
\hline MMV689060 & KINETOPLASTIDS & 95.8 & 3.8 & 92.2 & 0.3 \\
\hline MMV676191 & CRYPTOSPORIDIOSIS & 99.0 & 5.0 & 92.3 & 5.9 \\
\hline MMV024829 & MALARIA & 99.7 & 7.1 & 92.3 & 3.1 \\
\hline MMV676605 & MALARIA & 117.8 & 3.6 & 92.3 & 4.4 \\
\hline MMV1110498 & WOLBACHIA LF & 87.1 & 1.6 & 92.3 & 4.3 \\
\hline MMV676439 & TUBERCULOSIS & 108.0 & 5.0 & 92.3 & 8.3 \\
\hline MMV676597 & TUBERCULOSIS & 99.7 & 6.9 & 92.4 & 5.4 \\
\hline MMV688761 & SCHISTOSOMIASIS & 100.8 & 2.1 & 92.4 & 2.8 \\
\hline MMV688279 & KINETOPLASTIDS & 50.1 & 7.3 & 92.4 & 4.2 \\
\hline MMV676480 & ONCHOCERCIASIS & 93.0 & 7.8 & 92.5 & 8.1 \\
\hline MMV676382 & SCHISTOSOMIASIS & 92.1 & 7.8 & 92.6 & 6.9 \\
\hline MMV688796 & KINETOPLASTIDS & 90.3 & 2.0 & 92.8 & 2.8 \\
\hline MMV031011 & MALARIA & 119.0 & 13.1 & 92.8 & 19.3 \\
\hline MMV676395 & TUBERCULOSIS & 119.9 & 2.4 & 92.8 & 4.7 \\
\hline MMV687273 & TUBERCULOSIS & 94.4 & 9.4 & 92.9 & 9.9 \\
\hline MMV020623 & MALARIA & 100.0 & 7.8 & 93.1 & 6.1 \\
\hline MMV019234 & MALARIA & 95.8 & 4.6 & 93.1 & 4.4 \\
\hline MMV393144 & MALARIA & 89.8 & 4.0 & 93.1 & 3.9 \\
\hline MMV676528 & MALARIA & 127.2 & 3.9 & 93.1 & 6.2 \\
\hline MMV007638 & MALARIA & 96.6 & 4.7 & 93.2 & 2.1 \\
\hline MMV019807 & MALARIA & 96.7 & 3.5 & 93.3 & 4.4 \\
\hline MMV010545 & $\begin{array}{l}\text { MALARIA } \\
\text { REFERENCE }\end{array}$ & 93.1 & 4.3 & 93.3 & 5.0 \\
\hline MMV687798 & COMPOUNDS & 100.0 & 6.7 & 93.3 & 2.9 \\
\hline MMV688350 & DENGUE & 117.9 & 7.3 & 93.4 & 1.7 \\
\hline
\end{tabular}




\begin{tabular}{|c|c|c|c|c|c|}
\hline MMV687800 & $\begin{array}{l}\text { REFERENCE } \\
\text { COMPOUNDS }\end{array}$ & 24.0 & 2.4 & 93.4 & 10.0 \\
\hline MMV007920 & MALARIA & 90.0 & 2.5 & 93.5 & 2.2 \\
\hline MMV085210 & MALARIA & 112.4 & 2.4 & 93.5 & 5.4 \\
\hline MMV020152 & MALARIA & 62.9 & 19.2 & 93.6 & 5.6 \\
\hline MMV022236 & MALARIA & 86.3 & 9.0 & 93.7 & 6.7 \\
\hline MMV020391 & $\begin{array}{l}\text { MALARIA } \\
\text { REFERENCE }\end{array}$ & 101.5 & 3.1 & 93.8 & 0.7 \\
\hline MMV000011 & COMPOUNDS & 90.6 & 6.3 & 93.8 & 7.2 \\
\hline MMV099637 & KINETOPLASTIDS & 89.3 & 2.9 & 93.8 & 8.3 \\
\hline MMV676558 & TUBERCULOSIS & 46.7 & 4.9 & 93.9 & 7.5 \\
\hline MMV688548 & TOXOPLASMOSIS & 1.2 & 0.5 & 93.9 & 9.8 \\
\hline MMV1037162 & MALARIA & 97.2 & 13.6 & 94.0 & 16.3 \\
\hline MMV688271 & KINETOPLASTIDS & 92.7 & 3.4 & 94.2 & 2.5 \\
\hline MMV690103 & KINETOPLASTIDS & 96.1 & 9.1 & 94.2 & 9.7 \\
\hline MMV688776 & KINETOPLASTIDS & 96.4 & 8.1 & 94.3 & 6.1 \\
\hline MMV687188 & $\begin{array}{l}\text { TUBERCULOSIS } \\
\text { REFERENCE }\end{array}$ & 77.5 & 7.0 & 94.4 & 18.8 \\
\hline MMV000016 & COMPOUNDS & 77.5 & 7.0 & 94.4 & 18.8 \\
\hline MMV676461 & TUBERCULOSIS & 97.0 & 2.6 & 94.5 & 2.6 \\
\hline MMV045105 & KINETOPLASTIDS & 103.3 & 3.2 & 94.6 & 4.3 \\
\hline MMV1028806 & MALARIA & 94.7 & 1.3 & 94.7 & 3.1 \\
\hline MMV676409 & TUBERCULOSIS & 86.7 & 7.5 & 94.8 & 8.5 \\
\hline MMV688793 & KINETOPLASTIDS & 103.2 & 3.3 & 94.8 & 4.1 \\
\hline MMV006372 & $\begin{array}{l}\text { MALARIA } \\
\text { REFERENCE }\end{array}$ & 101.5 & 12.2 & 95.0 & 13.5 \\
\hline MMV000063 & COMPOUNDS & 93.7 & 6.1 & 95.1 & 5.8 \\
\hline MMV020512 & MALARIA & 93.1 & 1.3 & 95.1 & 7.1 \\
\hline MMV001059 & MALARIA & 88.1 & 1.9 & 95.2 & 4.1 \\
\hline MMV016136 & MALARIA & 88.4 & 11.8 & 95.2 & 5.3 \\
\hline MMV676877 & MALARIA & 93.4 & 1.3 & 95.4 & 2.0 \\
\hline MMV688416 & DENGUE & 103.5 & 7.6 & 95.5 & 7.5 \\
\hline MMV000907 & MALARIA & 109.4 & 10.3 & 95.5 & 6.2 \\
\hline MMV676048 & KINETOPLASTIDS & 113.9 & 11.6 & 95.5 & 14.7 \\
\hline MMV688470 & TOXOPLASMOSIS & 97.4 & 9.2 & 95.6 & 4.6 \\
\hline MMV021057 & MALARIA & 96.1 & 8.5 & 95.6 & 6.3 \\
\hline MMV676444 & TUBERCULOSIS & 93.2 & 3.0 & 95.7 & 3.7 \\
\hline MMV688980 & MALARIA & 110.5 & 7.0 & 95.7 & 2.3 \\
\hline MMV676520 & TUBERCULOSIS & 100.7 & 5.8 & 95.7 & 10.2 \\
\hline MMV688364 & TOXOPLASMOSIS & 100.2 & 5.9 & 95.7 & 1.6 \\
\hline MMV688474 & $\begin{array}{l}\text { KINETOPLASTIDS } \\
\text { LYMPHATIC }\end{array}$ & 84.0 & 3.1 & 95.8 & 1.7 \\
\hline MMV687776 & $\begin{array}{l}\text { FILARIASIS } \\
\text { REFERENCE }\end{array}$ & 108.2 & 6.4 & 95.8 & 3.0 \\
\hline MMV003152 & COMPOUNDS & 98.1 & 3.8 & 95.8 & 2.7 \\
\hline MMV676604 & KINETOPLASTIDS & 99.2 & 3.4 & 95.9 & 7.1 \\
\hline
\end{tabular}




\begin{tabular}{|c|c|c|c|c|c|}
\hline MMV688936 & TUBERCULOSIS & 88.1 & 1.6 & 95.9 & 4.4 \\
\hline MMV001493 & $\begin{array}{l}\text { ONCHOCERCIASIS } \\
\text { REFERENCE }\end{array}$ & 95.8 & 2.0 & 95.9 & 5.4 \\
\hline MMV000062 & COMPOUNDS & 96.0 & 9.5 & 96.0 & 4.0 \\
\hline MMV553002 & TUBERCULOSIS & 92.7 & 5.9 & 96.0 & 8.3 \\
\hline MMV202553 & KINETOPLASTIDS & 87.5 & 8.2 & 96.2 & 5.3 \\
\hline MMV687246 & MALARIA & 102.6 & 2.9 & 96.4 & 5.6 \\
\hline MMV687172 & TUBERCULOSIS & 117.1 & 5.8 & 96.4 & 8.9 \\
\hline MMV020120 & MALARIA & 95.5 & 1.3 & 96.5 & 3.0 \\
\hline MMV688763 & SCHISTOSOMIASIS & 92.8 & 2.8 & 96.6 & 2.4 \\
\hline MMV689061 & KINETOPLASTIDS & 8.4 & 5.8 & 96.7 & 3.7 \\
\hline MMV687706 & KINETOPLASTIDS & 100.9 & 1.3 & 96.8 & 2.0 \\
\hline MMV020136 & MALARIA & 107.2 & 7.5 & 96.8 & 2.8 \\
\hline MMV676512 & TUBERCULOSIS & 94.2 & 2.3 & 96.8 & 4.1 \\
\hline MMV661713 & TUBERCULOSIS & 12.6 & 2.5 & 97.0 & 3.3 \\
\hline MMV676411 & TUBERCULOSIS & 114.2 & 7.0 & 97.1 & 13.1 \\
\hline MMV019087 & MALARIA & 77.1 & 10.0 & 97.1 & 3.4 \\
\hline MMV676186 & KINETOPLASTIDS & 93.7 & 2.0 & 97.6 & 20.6 \\
\hline MMV024035 & MALARIA & 92.6 & 9.7 & 97.8 & 4.4 \\
\hline MMV676161 & KINETOPLASTIDS & 115.3 & 1.9 & 97.9 & 2.4 \\
\hline MMV671636 & ONCHOCERCIASIS & 108.9 & 1.3 & 98.0 & 4.5 \\
\hline MMV020321 & MALARIA & 93.1 & 3.6 & 98.0 & 3.6 \\
\hline MMV047015 & TUBERCULOSIS & 90.7 & 8.6 & 98.1 & 3.3 \\
\hline MMV676474 & TUBERCULOSIS & 104.3 & 5.9 & 98.2 & 1.6 \\
\hline MMV688554 & TUBERCULOSIS & 107.4 & 14.3 & 98.2 & 9.6 \\
\hline MMV688553 & TUBERCULOSIS & 102.6 & 7.7 & 98.2 & 7.0 \\
\hline MMV011511 & MALARIA & 108.7 & 9.3 & 98.3 & 10.4 \\
\hline MMV676600 & KINETOPLASTIDS & 109.7 & 4.9 & 98.3 & 7.5 \\
\hline MMV069458 & $\begin{array}{l}\text { TUBERCULOSIS } \\
\text { REFERENCE }\end{array}$ & 100.2 & 1.1 & 98.5 & 1.0 \\
\hline MMV000023 & COMPOUNDS & 92.6 & 2.4 & 98.5 & 2.6 \\
\hline MMV689255 & CRYPTOSPORIDIOSIS & 101.7 & 1.7 & 98.6 & 2.1 \\
\hline MMV026020 & MALARIA & 97.2 & 0.7 & 98.7 & 1.9 \\
\hline MMV676063 & ONCHOCERCIASIS & 113.4 & 3.5 & 98.7 & 3.8 \\
\hline MMV024114 & MALARIA & 96.4 & 9.2 & 98.8 & 9.3 \\
\hline MMV020982 & MALARIA & 100.2 & 3.3 & 98.8 & 6.6 \\
\hline MMV688768 & SCHISTOSOMIASIS & 85.2 & 10.9 & 98.9 & 2.8 \\
\hline MMV688934 & $\begin{array}{l}\text { KINETOPLASTIDS } \\
\text { REFERENCE }\end{array}$ & 97.6 & 10.2 & 99.3 & 2.1 \\
\hline MMV689758 & COMPOUNDS & 105.2 & 9.2 & 99.4 & 13.8 \\
\hline MMV687762 & KINETOPLASTIDS & 85.8 & 8.9 & 99.4 & 9.3 \\
\hline MMV053220 & TUBERCULOSIS & 89.9 & 15.6 & 99.6 & 8.2 \\
\hline MMV676379 & TUBERCULOSIS & 95.2 & 6.4 & 99.7 & 9.7 \\
\hline MMV007625 & MALARIA & 55.6 & 27.5 & 99.7 & 24.6 \\
\hline MMV675968 & CRYPTOSPORIDIOSIS & 101.1 & 5.2 & 99.8 & 9.5 \\
\hline
\end{tabular}




\begin{tabular}{|c|c|c|c|c|c|}
\hline MMV688178 & SCHISTOSOMIASIS & 104.3 & 15.6 & 99.8 & 8.1 \\
\hline MMV676064 & ONCHOCERCIASIS & 106.8 & 7.9 & 99.9 & 2.7 \\
\hline MMV676260 & MALARIA & 102.4 & 4.7 & 99.9 & 3.3 \\
\hline MMV676445 & TUBERCULOSIS & 103.7 & 11.5 & 99.9 & 6.8 \\
\hline MMV011691 & MALARIA & 109.3 & 8.9 & 100.0 & 1.6 \\
\hline MMV009135 & MALARIA & 108.0 & 3.0 & 100.1 & 5.5 \\
\hline MMV676539 & TUBERCULOSIS & 102.7 & 3.2 & 100.1 & 2.3 \\
\hline MMV102872 & TUBERCULOSIS & 94.6 & 5.3 & 100.1 & 9.1 \\
\hline MMV006901 & MALARIA & 4.8 & 1.4 & 100.1 & 19.9 \\
\hline MMV020670 & MALARIA & 97.2 & 3.2 & 100.1 & 5.0 \\
\hline MMV668727 & ONCHOCERCIASIS & 111.0 & 2.5 & 100.3 & 3.8 \\
\hline MMV006741 & MALARIA & 103.4 & 8.3 & 100.5 & 2.9 \\
\hline MMV022029 & $\begin{array}{l}\text { MALARIA } \\
\text { REFERENCE }\end{array}$ & 92.1 & 3.4 & 100.6 & 8.3 \\
\hline MMV687803 & COMPOUNDS & 109.4 & 5.2 & 100.7 & 3.9 \\
\hline MMV023953 & MALARIA & 94.9 & 7.0 & 101.3 & 5.6 \\
\hline MMV202458 & TUBERCULOSIS & 103.5 & 8.3 & 101.8 & 3.7 \\
\hline MMV676401 & TUBERCULOSIS & 106.4 & 10.2 & 101.9 & 11.4 \\
\hline MMV688555 & TUBERCULOSIS & 81.6 & 14.3 & 102.0 & 17.7 \\
\hline MMV676603 & TUBERCULOSIS & 105.5 & 5.6 & 102.6 & 2.8 \\
\hline MMV688888 & TUBERCULOSIS & 104.6 & 14.7 & 103.3 & 20.4 \\
\hline MMV688362 & KINETOPLASTIDS & 114.7 & 22.2 & 103.5 & 20.4 \\
\hline MMV676398 & WOLBACHIA LF & 108.2 & 9.9 & 104.1 & 8.0 \\
\hline MMV688407 & KINETOPLASTIDS & 102.9 & 4.3 & 104.1 & 14.5 \\
\hline MMV020291 & MALARIA & 102.3 & 6.6 & 104.4 & 5.2 \\
\hline MMV688941 & TUBERCULOSIS & 108.8 & 2.4 & 105.2 & 1.6 \\
\hline MMV020081 & MALARIA & 114.5 & 2.4 & 105.6 & 2.2 \\
\hline MMV026490 & MALARIA & 107.8 & 5.2 & 106.3 & 4.1 \\
\hline MMV676389 & TUBERCULOSIS & 94.8 & 2.9 & 107.1 & 3.9 \\
\hline MMV663250 & MALARIA & 115.6 & 5.3 & 107.4 & 9.4 \\
\hline MMV676881 & MALARIA & 103.5 & 5.6 & 107.8 & 9.8 \\
\hline MMV024101 & MALARIA & 112.6 & 15.0 & 108.2 & 7.1 \\
\hline MMV010576 & MALARIA & 100.6 & 15.6 & 108.5 & 8.5 \\
\hline MMV032967 & MALARIA & 109.8 & 1.6 & 108.6 & 3.0 \\
\hline MMV687812 & TUBERCULOSIS & 110.2 & 2.2 & 110.8 & 4.0 \\
\hline MMV688469 & TOXOPLASMOSIS & 101.7 & 4.2 & 110.8 & 3.2 \\
\hline MMV007133 & MALARIA & 112.0 & 5.9 & 110.8 & 3.8 \\
\hline MMV084603 & MALARIA & 97.0 & 2.1 & 111.5 & 5.5 \\
\hline MMV688180 & KINETOPLASTIDS & 108.1 & 19.7 & 116.2 & 19.5 \\
\hline MMV022478 & MALARIA & 94.7 & 5.1 & 116.9 & 6.0 \\
\hline MMV023388 & MALARIA & 140.1 & 2.7 & 117.3 & 13.3 \\
\hline
\end{tabular}




\section{Table S2}

Table S2. Cytotoxicity data $\left(\mathrm{CC}_{50}\right.$ values) for selected 'hit' compounds. Data is obtained either from the MMV Pathogen Box website (see citations) or experimentally. Shown are mean $\mathrm{CC}_{50}{ }^{72 \mathrm{~h}}$ values $(\mathrm{nM})$ calculated from three biological replicates $\pm \mathrm{SE}$.

\begin{tabular}{|c|c|c|}
\hline Compound & \multicolumn{2}{|c|}{ Cytotoxicity, $\mathrm{CC}_{50} \pm \mathrm{SE}(\mathrm{nM})$} \\
\hline & RAW 264.7ª & $\begin{array}{lr}\text { ^HeLa } & \text { Cells } \\
{ }^{*} \mathrm{HepG} 2 /{ }^{*} \mathrm{MRC5}{ }^{\mathrm{b}}\end{array}$ \\
\hline MMV676412 & $1600 \pm 120$ & ${ }^{*} 2200$ \\
\hline MMV676477 & $2200 \pm 410$ & ${ }^{*} 1300 / \wedge 2600$ \\
\hline MMV688372 & $>10000$ & *30000 \\
\hline MMV652003 & $8200 \pm 200$ & \#32000 \\
\hline MMV595321 & $>10000$ & $\# 6600$ \\
\hline MMV090930 & ND & *47000 \\
\hline
\end{tabular}

a Data obtained as part of this study.

${ }^{b}$ Data provided with the MMV Pathogen Box.

*Data obtained by MMV against HepG2.

\# Data obtained by MMV against MRC5.

^ Data obtained from the literature [70]. 